\title{
Performance Measurement of Internet Service
}

\section{Sigit Haryadi}

Institut Teknologi Bandung

March 2018

\section{Paper DOI10.17605/OSF.IO/8EXMU}

Citation: Haryadi, S. (2018, March 5). Performance Measurement of Internet Service. Retrieved from osf.io/preprints/inarxiv/8exmu

\section{Abstract}

This paper summarizes my papers and books, which reports on studies concerning the performance measurement of Internet services, particularly on mobile networks. In detail, Internet service quality measurements are grouped into two, the first is a measurement that aims to measure the provider's customer satisfaction, performed by the provider or by the user, and the second measurement goal is that the provider and/or by the vendor in relation to the quality assurance provided by the vendor to the provider. In detail, the first type of measurement uses a sampling technique and uses only one performance indicator, i.e. the data rate, otherwise, the second type performs the aggregate measurements with references to the signaling ladder diagram of the Internet services and measures many performance indicators.

\section{Types of Quality of Experience and Quality of Service Measurement of Internet Service}

Measurement of internet service quality is grouped into two, the first is a measurement that aims to measure the provider's customer satisfaction and the second measurement goal is that the provider and/or vendor is involved with the quality assurance provided by the vendor to the provider. 


\section{Quality Measurement of Internet Service for Customer Satisfaction}

In relation to the customer satisfaction, internet service quality measurements are carried out in a sampling of customers of the providers, thus the measured is QoE (Quality of Experience) rather than QoS (Quality of Service). In this type of measurement, the measured parameter is the data rate. Unlike the QoE measurements of telephone and chat services, where the Quality of Experience is shown in the form of Mean Opinion Score (MOS), otherwise, the measurement of the mean data rate uses units of kbps or Mbps.

The application of statistical science should be accompanied by an in-depth understanding of the customer characteristics of the service provider, where the number of respondents interviewed is generally much more than the amount required for data processing.

For example, we only need data to be processed as many as 100 customers, then we should have 100 customers who meet all the criteria of the type of customer provider, assume that is that meet the criteria for comparison of female and male customers, e.g. $55 \%$ versus $45 \%$, and also meet the customer age criteria, e.g. $60 \%$ of youth and $40 \%$ of established age, also meet the criteria of income level, e.g. $65 \%$ lower and $35 \%$ middle, and should meeting the criteria of call intensity, e.g. $75 \%$ rarely call and $25 \%$ more than three times a day.

There are two ways of reporting the measurement results of the data rate, first reporting the mean data rate with the standard deviation, and secondly reporting the minimum data rate reached by $90 \%$ of customers.

\section{Quality Measurement of the Internet Services in Relation between Provider and Vendor or OTT Provider.}

Measurements of network performance and quality of Internet services in relation to vendors should be done based on the aggregate data, not a sampling, and should refer to the Signaling Ladder Diagram of the Internet services. The problem faced by the provider is almost the 
vendor's Signaling Ladder Diagram is not $100 \%$ the same as the Signaling Ladder Diagram compiled by International Standard ETSI and 3 GPP. Another problem is related to services provided by over-the-top providers that have no interconnection links with broadband providers, so that providers cannot ask for quality accountability to over-the-top providers. Some important parameters on this type of measurement are:

1) Mean Data Rate or Minimum Data Rate

2) Internet Protocol Service non-accessibility

3) Internet Protocol Service Access Failure Ratio

4) Internet Protocol Service Access Time

5) Session Failure Ratio

6) Internet Protocol Setup times

7) Session times

8) Data Transfer Cut-off Ratio

9) Teardown Failure Ratio

10) Teardown Time

\subsection{Measurement of Mean or Minimum Data Rate}

Mean data rate parameter shows the value of the average speed of the data transmission, where average throughput values calculated from the start to build a data connection until the whole data is received successfully. Accompanying the mean data rate report, it is important to report also the standard deviation.

The minimum data rate parameter shows $90 \%$ of customers with the fastest data rate, which may also take a percentage larger or smaller than $90 \%$.

Pre-condition for this parameter is UE has managed to attach, the connection has been active PDP context, as well as the service has been successfully carried out. 
Abstract Equation of Internet Data rate:

$$
\text { Data rate }\left(\frac{k b i t}{s}\right)=\frac{\text { User Data Transferred }(k b i t)}{t_{\text {data transfer complete }} t_{\text {start of data transfer }}}
$$

\subsection{Measurement of Internet Service Non Accessibility}

This parameter indicates the probability of the UE (User Equipment) can not establish a PDP context and can not access the internet access.

Pre condition for this parameter is that the UE has managed to make the process of attachment. Abstract Equation of Internet Protocol Service Non-Accessibility:

Internet Service Non Accessiblity $(\%)=\frac{\text { Unsuccessful Attempts to Reach the Point when contents is reveived }}{\text { All Attemptes to reach the Point when contents is received } 100}$

\subsection{Measurement of Internet Service Access Failure Ratio}

This parameter indicates the probability of an UE cannot successfully establish the TCP/IP connection to the server of the service.

Pre condition for this parameter is UE has managed to make the process of attachment and connection PDP context is active.

Abstract Equation of Internet Service Access Failure:

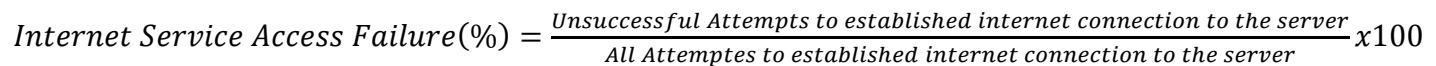

\subsection{Measurement of Internet Service Access Time}

This parameter represents the time required for the UE establish TCP/IP connection to the server from a service successfully.

Pre-condition for this parameter is UE has managed to attach and connection PDP context is active, so that the parameter can only be calculated on successful trials.

Abstract Equation of Internet Service Access Time:

Internet Service Access Time (second) $=t_{\text {Reveption of the Furst of packet data }}-t_{\text {Service Request }}$

\subsection{Measurement of Session Failure Ratio}

This parameter shows the ratio of a successful session until the normal end of the service. 
Pre condition for this parameter is UE has managed to attach and connection PDP context is active.

Abstract Equation of Session Failure Ratio:

$$
\text { Session Failure ratio }(\%)=\frac{\text { Uncomplete Sessions }}{\text { Successful Started Sessions }} \times 100
$$

\subsection{Measurement of Internet Protocol Setup times}

This parameter represents the time required to access the full service, from establishing PDP context until receiving the first data packet.

Pre-condition for this parameter is UE has managed to make the process of attachment.

Abstract Equation of Internet Protocol Service Setup Time:

Internet Service Setup Time $($ second $)=t_{\text {the Content had been sent or Received }}-t_{\text {the Connection had been Initiated }}$

\subsection{Measurement of Session times}

This parameter shows the time required to process the data sessions to successfully complete.

Pre condition for this parameter is UE has managed to attach and connection PDP context is active.

Abstract Equation of Internet Protocol Service Setup Time:

$$
\text { Session Time }(\text { second })=t_{\text {the End of Session }}-t_{\text {Session Start }}
$$

\subsection{Measurement of Data Transfer Cut-off ratio}

This parameter shows the ratio between the incomplete data transfer and data transfer begins with success.

Pre-condition for this parameter is UE has managed to attachment and connection PDP context is active.

Abstract Equation of Data Transfer Cut-off Ratio (\%):

$$
\text { Data Transfer Ratio(\%) }=\frac{\text { Incomplete Data Transfer }}{\text { Successful Sarted Sessions }} \times 100
$$




\subsection{Measurement of Teardown Failure Ratio (\%)}

Probability that the RTSP message (Real-Time Streaming Protocol message) or the

"Teardown" message is sent from the UE to the server and there is no RTSP response "200

$\mathrm{OK}^{\prime \prime}$ is received from the EU server.

Abstract Equation of Teardown Failure Ratio (\%):

$$
\text { Teardown Failure Ratio(\%) }=\frac{\text { Number of Cases Without the } 200 \text { OK Response }}{\text { All Teardown Attempt by UE }} \times 100
$$

\subsection{Measurement of Teardown Time}

Duration of access to services measures from demand streaming or streaming requests on the portal to the reception of the first stream of data packets to the UE

Abstract Equation of Teardown Time:

$$
\text { Teardown Time }(\text { second })=t_{\text {Server Response to Teardown Message }}-t_{U E \text { Sending Teardown Message }}
$$

\section{Signaling Ladder Diagram}

The following is a list of Signaling Ladder Diagrams that are essential for measuring the quality of Internet services in an aggregate, most of which are referred to Internet Forum standards, and a small portion of Signaling Ladder Diagrams is referring to ETSI standards.

\begin{tabular}{|c|c|c|c|c|}
\hline Trigger Point & $\begin{array}{l}\text { Logical } \\
\text { Channel }\end{array}$ & Signal Name & $\begin{array}{l}\text { Outgoing Network } \\
\text { Element }\end{array}$ & $\begin{array}{l}\text { Incoming Network } \\
\text { Element }\end{array}$ \\
\hline \multirow{5}{*}{$\begin{array}{l}\text { START: Reception of the } \\
\text { first data } \\
\text { packet which contain the } \\
\text { content. }\end{array}$} & & HTTP OK 200 & $\begin{array}{ll}\text { HTTP } & \text { WEB } \\
\text { SERVER } & \end{array}$ & GATEWAY \\
\hline & & HTTP OK 200 & GATEWAY & MS \\
\hline & & Ack & MS & GATEWAY \\
\hline & & HTTP Continue & $\begin{array}{l}\text { HTTP } \\
\text { SERVER }\end{array}$ & MS \\
\hline & & Ack & MS & $\begin{array}{ll}\text { HTTP } & \text { WEB } \\
\text { SERVER }\end{array}$ \\
\hline $\begin{array}{l}\text { STOP: Reception of the last } \\
\text { data packet which } \\
\text { contain the content. }\end{array}$ & & HTTP Continue & $\begin{array}{l}\text { HTTP } \\
\text { SERVER }\end{array}$ & MS \\
\hline
\end{tabular}

Table 1. Signaling Ladder Diagram of HTTP/Web Browsing Data Rate 
The process of web browsing mean data rate measurement can be started from the reception of the first data packet containing content, marked by sending a "200 OK" signal from web server to Gateway until the reception of the last data packet containing content, marked by sending a "HTTP continue" signal from web server to MS.

Table 2. Signaling Ladder Diagram of Internet Service Non-Accessibility in 3G Network

\begin{tabular}{|c|c|c|c|c|}
\hline Trigger Point & $\begin{array}{l}\text { Logical } \\
\text { Channel }\end{array}$ & Signal Name & $\begin{array}{l}\text { Outgoing } \\
\text { Network Element }\end{array}$ & $\begin{array}{l}\text { Incoming } \\
\text { Network Element }\end{array}$ \\
\hline \multirow{25}{*}{$\begin{array}{l}\text { START: ATD } \\
\text { command. }\end{array}$} & & Activate PDP Context & MS & SGSN \\
\hline & & $\begin{array}{l}\text { Create PDP Context } \\
\text { Request }\end{array}$ & SGSN & GGSN \\
\hline & & $\begin{array}{l}\text { Create PDP Context } \\
\text { Response }\end{array}$ & GGSN & SGSN \\
\hline & & $\begin{array}{l}\text { Radio Access Bearer } \\
\text { Assignment Request }\end{array}$ & 3G RAN & SGSN \\
\hline & & Radio Bearer Setup & 3G RAN & MS \\
\hline & & $\begin{array}{l}\text { Radio Bearer Setup } \\
\text { Complete }\end{array}$ & MS & 3G RAN \\
\hline & & $\begin{array}{l}\text { Radio Access Bearer } \\
\text { Assignment Response }\end{array}$ & 3G RAN & SGSN \\
\hline & & $\begin{array}{l}\text { Update PDP Context } \\
\text { Request }\end{array}$ & SGSN & GGSN \\
\hline & & $\begin{array}{l}\text { Update PDP Context } \\
\text { Response }\end{array}$ & GGSN & SGSN \\
\hline & & $\begin{array}{l}\text { Activate PDP Context } \\
\text { Accept }\end{array}$ & SGSN & MS \\
\hline & & $\begin{array}{lll}\text { Update } & \text { PDP } & \text { Context } \\
\text { Request } & & \end{array}$ & SGSN & GGSN \\
\hline & & $\begin{array}{l}\text { Update PDP Context } \\
\text { Response }\end{array}$ & GGSN & SGSN \\
\hline & & $\begin{array}{l}\text { Radio Access Bearer } \\
\text { Modification }\end{array}$ & MS & 3G RAN \\
\hline & & $\begin{array}{l}\text { Radio Access Bearer } \\
\text { Modification }\end{array}$ & 3G RAN & MS \\
\hline & & $\begin{array}{l}\text { Radio Access Bearer } \\
\text { Modification }\end{array}$ & 3G RAN & SGSN \\
\hline & & $\begin{array}{l}\text { Radio Access Bearer } \\
\text { Modification }\end{array}$ & SGSN & 3G RAN \\
\hline & & $\begin{array}{l}\text { Modify PDP Context } \\
\text { Request }\end{array}$ & SGSN & MS \\
\hline & & $\begin{array}{lll}\text { Modify } & \text { PDP Context } \\
\text { Accept } & & \\
\end{array}$ & MS & SGSN \\
\hline & & Invoke Trace & SGSN & 3G RAN \\
\hline & & SYN & MS & GATEWAY \\
\hline & & SYN, ACK & GATEWAY & MS \\
\hline & & ACK & MS & GATEWAY \\
\hline & & HTTP GET & MS & GATEWAY \\
\hline & & DNS QUERY & GATEWAY & DNS SERVER \\
\hline & & DNS REPLY & DNS SERVER & GATEWAY \\
\hline
\end{tabular}




\begin{tabular}{|c|c|c|c|}
\hline & SYN & GATEWAY & $\begin{array}{l}\text { HTTP } \\
\text { SERVER }\end{array}$ \\
\hline & SYN, ACK & $\begin{array}{ll}\text { HTTP } & \text { WEB } \\
\text { SERVER } & \end{array}$ & GATEWAY \\
\hline & ACK & GATEWAY & $\begin{array}{l}\text { HTTP } \\
\text { SERVER }\end{array}$ \\
\hline & HTTP GET & GATEWAY & $\begin{array}{l}\text { HTTP } \\
\text { SERVER }\end{array}$ \\
\hline & Ack & $\begin{array}{l}\text { HTTP } \\
\text { SERVER }\end{array}$ & GATEWAY \\
\hline & Ack & GATEWAY & MS \\
\hline & HTTP OK 200 & $\begin{array}{l}\text { HTTP } \\
\text { SERVER }\end{array}$ & GATEWAY \\
\hline $\begin{array}{l}\text { STOP: Reception of } \\
\text { the first data } \\
\text { packet which } \\
\text { contain the content. }\end{array}$ & HTTP OK 200 & GATEWAY & MS \\
\hline
\end{tabular}

Table 3 Signaling Ladder Diagram of Internet Service Setup Time

\begin{tabular}{|c|c|c|c|c|}
\hline Trigger Point & $\begin{array}{l}\text { Logical } \\
\text { Channel }\end{array}$ & $\begin{array}{l}\text { Signal } \\
\text { Name }\end{array}$ & $\begin{array}{l}\text { Outgoing Network } \\
\text { Element }\end{array}$ & $\begin{array}{l}\text { Incoming Network } \\
\text { Element }\end{array}$ \\
\hline \multirow{13}{*}{$\begin{array}{l}\text { START: the First [SYN] } \\
\text { message sent }\end{array}$} & & SYN & MS & GATEWAY \\
\hline & & SYN, ACK & GATEWAY & MS \\
\hline & & ACK & MS & GATEWAY \\
\hline & & HTTP GET & MS & GATEWAY \\
\hline & & $\begin{array}{l}\text { DNS } \\
\text { QUERY }\end{array}$ & GATEWAY & DNS SERVER \\
\hline & & $\begin{array}{l}\text { DNS } \\
\text { REPLY }\end{array}$ & DNS SERVER & GATEWAY \\
\hline & & SYN & GATEWAY & HTTP WEB SERVER \\
\hline & & SYN, ACK & POP3 SERVER & GATEWAY \\
\hline & & ACK & GATEWAY & HTTP WEB SERVER \\
\hline & & HTTP GET & GATEWAY & HTTP WEB SERVER \\
\hline & & Ack & HTTP WEB SERVER & GATEWAY \\
\hline & & Ack & GATEWAY & MS \\
\hline & & $\begin{array}{l}\text { HTTP OK } \\
200\end{array}$ & HTTP WEB SERVER & GATEWAY \\
\hline $\begin{array}{l}\text { STOP: Reception of the } \\
\text { first data } \\
\text { packet which contain the } \\
\text { content }\end{array}$ & & $\begin{array}{l}\text { HTTP OK } \\
200\end{array}$ & GATEWAY & MS \\
\hline
\end{tabular}

HTTP services setup time in $3 \mathrm{G}$ networks is measured beginning with the transmission of Synchronization signal by MS to Gateway, until the sending a HTTP 200 OK signal by Gateway to MS.

Table 4. Signaling Ladder Diagram of Internet Service Access Success Rate

\begin{tabular}{|l|l|l|l|l|}
\hline Trigger Point & $\begin{array}{l}\text { Log } \\
\text { Ch }\end{array}$ & Signal Name & $\begin{array}{l}\text { Outgoing Network } \\
\text { Element }\end{array}$ & $\begin{array}{l}\text { Incoming Network } \\
\text { Element }\end{array}$ \\
\hline $\begin{array}{l}\text { START: the First } \\
\text { [SYN] message sent }\end{array}$ & & SYN & MS & GATEWAY \\
\hline & & SYN, ACK & GATEWAY & MS \\
\hline & & ACK & MS & GATEWAY \\
\hline
\end{tabular}




\begin{tabular}{|l|l|l|l|}
\hline & HTTP GET & MS & GATEWAY \\
\hline & DNS QUERY & GATEWAY & DNS SERVER \\
\hline & DNS REPLY & DNS SERVER & GATEWAY \\
\hline & SYN & GATEWAY & HTTP WEB SERVER \\
\hline & SYN, ACK & POP3 SERVER & GATEWAY \\
\hline & ACK & GATEWAY & HTTP WEB SERVER \\
\hline & HTTP GET & GATEWAY & HTTP WEB SERVER \\
\hline & Ack & HTTP WEB SERVER & GATEWAY \\
\hline & Ack & GATEWAY & MS \\
\hline & HTTP OK 200 & HTTP WEB SERVER & GATEWAY \\
\hline $\begin{array}{l}\text { STOP: Reception of } \\
\text { the first data } \\
\text { packet which contain } \\
\text { the content }\end{array}$ & HTTP OK 200 & GATEWAY & MS \\
\hline
\end{tabular}

HTTP services success access rate in $3 \mathrm{G}$ networks, beginning with the transmission of Synchronization signal by MS to Gateway, until the sending a HTTP 200 OK signal by Gateway to MS.

Table 5. Signaling Ladder Diagram of FTP Download Service

\begin{tabular}{|c|c|c|c|c|}
\hline Trigger Point & $\begin{array}{l}\text { Logical } \\
\text { Channel }\end{array}$ & Signal Name & $\begin{array}{l}\text { Outgoing } \\
\text { Network } \\
\text { Element }\end{array}$ & $\begin{array}{l}\text { Incoming } \\
\text { Network } \\
\text { Element }\end{array}$ \\
\hline START: ATD & & Activate PDP Context & MS & SGSN \\
\hline & & Create PDP Context Request & SGSN & GGSN \\
\hline & & Create PDP Context Response & GGSN & SGSN \\
\hline & & $\begin{array}{l}\text { Radio Access Bearer Assignment } \\
\text { Request }\end{array}$ & 3G RAN & SGSN \\
\hline & & Radio Bearer Setup & 3G RAN & MS \\
\hline & & Radio Bearer Setup Complete & MS & 3G RAN \\
\hline & & $\begin{array}{l}\text { Radio Access Bearer Assignment } \\
\text { Response }\end{array}$ & 3G RAN & SGSN \\
\hline & & Update PDP Context Request & SGSN & GGSN \\
\hline & & Update PDP Context Response & GGSN & SGSN \\
\hline & & Activate PDP Context Accept & SGSN & MS \\
\hline & & Update PDP Context Request & SGSN & GGSN \\
\hline & & Update PDP Context Response & GGSN & SGSN \\
\hline & & $\begin{array}{l}\text { Radio Access Bearer } \\
\text { Modification }\end{array}$ & MS & 3G RAN \\
\hline & & $\begin{array}{l}\text { Radio Access Bearer } \\
\text { Modification }\end{array}$ & 3G RAN & MS \\
\hline & & $\begin{array}{l}\text { Radio Access Bearer } \\
\text { Modification }\end{array}$ & 3G RAN & SGSN \\
\hline & & $\begin{array}{l}\text { Radio Access Bearer } \\
\text { Modification }\end{array}$ & SGSN & 3G RAN \\
\hline & & Modify PDP Context Request & SGSN & MS \\
\hline & & Modify PDP Context Accept & MS & SGSN \\
\hline & & Invoke Trace & SGSN & 3G RAN \\
\hline & & SYN & MS & GATEWAY \\
\hline & & SYN, ACK & GATEWAY & MS \\
\hline & & ACK & MS & GATEWAY \\
\hline & & Connect & MS & GATEWAY \\
\hline
\end{tabular}




\begin{tabular}{|c|c|c|c|}
\hline & DNS QUERY & GATEWAY & DNS SERVER \\
\hline & DNS Response & DNS SERVER & GATEWAY \\
\hline & SYN & GATEWAY & FTP SERVER \\
\hline & SYN, ACK & FTP SERVER & GATEWAY \\
\hline & ACK & GATEWAY & FTP SERVER \\
\hline & Connect & GATEWAY & FTP SERVER \\
\hline & Ack & FTP SERVER & MS \\
\hline & FTP220 & FTP SERVER & MS \\
\hline & Ack & MS & FTP SERVER \\
\hline & FTP331 & FTP SERVER & MS \\
\hline & Ack & MS & FTP SERVER \\
\hline & Send username and password & MS & FTP SERVER \\
\hline & Ack & FTP SERVER & MS \\
\hline & FTP 230 & FTP SERVER & MS \\
\hline & Ack & MS & FTP SERVER \\
\hline & FTP150 & FTP SERVER & MS \\
\hline & SYN & FTP SERVER & MS \\
\hline & SYN, ACK & MS & FTP SERVER \\
\hline & Ack & FTP SERVER & MS \\
\hline & FTP200 & FTP SERVER & MS \\
\hline & Ack & MS & FTP SERVER \\
\hline & LIST & MS & FTP SERVER \\
\hline & Ack & FTP SERVER & MS \\
\hline & FTP 226 & MS & FTP SERVER \\
\hline & Ack & FTP SERVER & MS \\
\hline & FTP GET & MS & FTP SERVER \\
\hline & Ack & FTP SERVER & MS \\
\hline & RETR & MS & FTP SERVER \\
\hline & Ack & FTP SERVER & MS \\
\hline & FTP150 & FTP SERVER & MS \\
\hline & SYN & FTP SERVER & MS \\
\hline & SYN, ACK & MS & FTP SERVER \\
\hline & Ack & FTP SERVER & MS \\
\hline $\begin{array}{l}\text { STOP: Reception } \\
\text { of the first data } \\
\text { packet which } \\
\text { contain the } \\
\text { content. }\end{array}$ & FTP DATA & FTP SERVER & MS \\
\hline
\end{tabular}

The process of FTP download measurement can be started from the ATD Command, marked by sending an Activate PDP Context signal from MS to SGSN until the reception of the first data packet containing content, marked by sending a FTP Data signal from FTP server to MS. The process of FTP download measurement can be started from the ATD Command, marked by sending an Activate PDP Context signal from MS to SGSN until the reception of the first 
data packet containing content, marked by sending a FTP Data signal from FTP server to MS.

Table 6. Signaling Ladder Diagram of FTP Download

\begin{tabular}{|l|l|l|l|l|}
\hline Trigger Point & Logical Channel & Signal Name & $\begin{array}{l}\text { Outgoing } \\
\text { Network } \\
\text { Element }\end{array}$ & $\begin{array}{l}\text { Incoming } \\
\text { Network } \\
\text { Element }\end{array}$ \\
\hline $\begin{array}{l}\text { START: Reception } \\
\text { of the first data } \\
\text { packet which contain } \\
\text { the content. }\end{array}$ & FTP DATA & FTP SERVER & MS \\
\hline $\begin{array}{l}\text { STOP: Reception of } \\
\text { the last data packet } \\
\text { which } \\
\text { contain the content }\end{array}$ & Ack & MTP DATA + FIN & FTP SERVER & MS \\
\hline
\end{tabular}

The process of FTP download- mean data rate measurement can be started from the reception of the first data packet containing content, marked by sending a FTP Data signal from FTP server to MS until the reception of the last data packet containing content, marked by sending a FTP Data \& FIN signal from FTP server to MS.

Table 7. Signaling Ladder Diagram of "Ping" on 3G Network

\begin{tabular}{|c|c|c|c|c|c|}
\hline Trigger Point & $\begin{array}{l}\text { Logical } \\
\text { Channel }\end{array}$ & Signal Name & Abbreviation & $\begin{array}{l}\text { Outgoing } \\
\text { Network } \\
\text { Element }\end{array}$ & $\begin{array}{l}\text { Incoming } \\
\text { Network } \\
\text { Element }\end{array}$ \\
\hline \multirow{9}{*}{$\begin{array}{l}\text { START: UE or } \\
\text { MS sends the } \\
\text { first "Activate } \\
\text { PDP context } \\
\text { Request" } \\
\text { message (Layer } \\
\text { 3). }\end{array}$} & & Activate PDP Context & APC & MS & SGSN \\
\hline & & $\begin{array}{lll}\text { Create } & \text { PDP } & \text { Context } \\
\text { Request } & & \end{array}$ & CPCReq & SGSN & GGSN \\
\hline & & $\begin{array}{l}\text { Create PDP Context } \\
\text { Response }\end{array}$ & CPCRes & GGSN & SGSN \\
\hline & & $\begin{array}{l}\text { Radio Access Bearer } \\
\text { Assignment Request }\end{array}$ & RABAReq & 3G RAN & SGSN \\
\hline & & Radio Bearer Setup & RBS & 3G RAN & MS \\
\hline & & $\begin{array}{l}\text { Radio Bearer Setup } \\
\text { Complete }\end{array}$ & RBSCom & MS & 3G RAN \\
\hline & & $\begin{array}{l}\text { Radio Access Bearer } \\
\text { Assignment Response }\end{array}$ & RABARes & 3G RAN & SGSN \\
\hline & & $\begin{array}{lll}\text { Update } & \text { PDP } & \text { Context } \\
\text { Request } & & \end{array}$ & UPCReq & SGSN & GGSN \\
\hline & & $\begin{array}{l}\text { Update PDP Context } \\
\text { Response }\end{array}$ & UPCRes & GGSN & SGSN \\
\hline
\end{tabular}




\begin{tabular}{|c|c|c|c|}
\hline & Ack & MS & $\begin{array}{l}\text { MEDIA } \\
\text { SERVER }\end{array}$ \\
\hline & $\begin{array}{lll}\text { Activate } & \text { Secondary } & \text { PDP } \\
\text { Context } & & \end{array}$ & MS & SGSN \\
\hline & Create PDP Context Request & SGSN & GGSN \\
\hline & 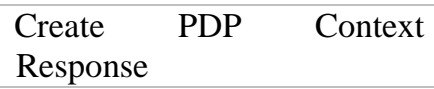 & GGSN & SGSN \\
\hline & $\begin{array}{l}\text { Radio Access Bearer } \\
\text { Assignment Request }\end{array}$ & 3G RAN & SGSN \\
\hline & Radio Bearer Setup & 3G RAN & MS \\
\hline & Radio Bearer Setup Complete & MS & 3G RAN \\
\hline & $\begin{array}{l}\text { Radio Access Bearer } \\
\text { Assignment Response }\end{array}$ & 3G RAN & SGSN \\
\hline & Update PDP Context Request & SGSN & GGSN \\
\hline & $\begin{array}{lll}\begin{array}{l}\text { Update } \\
\text { Response }\end{array} & \text { PDP } & \text { Context } \\
\end{array}$ & GGSN & SGSN \\
\hline & $\begin{array}{l}\text { Activate Secondary PDP } \\
\text { Context Accept }\end{array}$ & SGSN & MS \\
\hline & RTSP : Play & MS & $\begin{array}{l}\text { MEDIA } \\
\text { SERVER }\end{array}$ \\
\hline & Ack & $\begin{array}{l}\text { MEDIA } \\
\text { SERVER }\end{array}$ & MS \\
\hline & $200 \mathrm{OK}$ & $\begin{array}{l}\text { MEDIA } \\
\text { SERVER }\end{array}$ & MS \\
\hline & Ack & MS & $\begin{array}{l}\text { MEDIA } \\
\text { SERVER }\end{array}$ \\
\hline $\begin{array}{l}\text { STOP: Reception } \\
\text { of first data } \\
\text { packet. }\end{array}$ & IP/UDP/RTP & $\begin{array}{l}\text { MEDIA } \\
\text { SERVER }\end{array}$ & MS \\
\hline
\end{tabular}

The process of Streaming Service measurement can be started from by sending a synchronization signal from MS to Media Server until sending an IP/UDP/RTP signal from Media Server to MS.

Table 10. Signaling Ladder Diagram Streaming Reproduction

\begin{tabular}{|l|l|l|l|l|}
\hline Trigger Point & $\begin{array}{l}\text { Logical } \\
\text { Channel }\end{array}$ & Signal Name & $\begin{array}{l}\text { Outgoing } \\
\text { Element }\end{array}$ & $\begin{array}{l}\text { Network } \\
\text { Incoming } \\
\text { Network } \\
\text { Element }\end{array}$ \\
\hline $\begin{array}{l}\text { START: Streaming } \\
\text { player signals the } \\
\text { start of the } \\
\text { stream reproduction }\end{array}$ & & IP/UDP/RTP & MEDIA SERVER & MS \\
\hline & & IP/UDP/RTP & MEDIA SERVER & MS \\
\hline & RTCP & MS & $\begin{array}{l}\text { MEDIA } \\
\text { SERVER }\end{array}$ \\
\hline $\begin{array}{l}\text { STOP: RTSP } \\
\text { Teardown method } \\
\text { sent by UE and } \\
\text { reception of } \\
\text { confirmation "RTSP } \\
\text { 200 OK" from } \\
\text { media server. }\end{array}$ & RTSP : Teardown & MS & $\begin{array}{l}\text { MEDIA } \\
\text { SERVER }\end{array}$ \\
\hline & Ack & Ok 200 & MEDIA SERVER & MS \\
\hline
\end{tabular}


The process of Streaming Reproduction measurement can be started from by sending an IP/UDP/RTP from Media server to MS until sending an OK 200 signal from Media Server to MS.

Table 11. Signaling Ladder Diagram of E-Mail Login (Upload)

\begin{tabular}{|c|c|c|c|c|}
\hline Trigger Point & $\begin{array}{l}\text { Logical } \\
\text { Channel }\end{array}$ & Signal Name & $\begin{array}{l}\text { Outgoing Network } \\
\text { Element }\end{array}$ & $\begin{array}{l}\text { Incoming Network } \\
\text { Element }\end{array}$ \\
\hline \multirow{20}{*}{$\begin{array}{l}\text { START: TCP: } \\
\text { First "SYN" sent by } \\
\text { the client }\end{array}$} & & SYN & MS & GATEWAY \\
\hline & & SYN, ACK & GATEWAY & MS \\
\hline & & ACK & MS & GATEWAY \\
\hline & & Connect & MS & GATEWAY \\
\hline & & DNS QUERY & GATEWAY & DNS SERVER \\
\hline & & DNS Response & DNS SERVER & GATEWAY \\
\hline & & SYN & GATEWAY & SMTP SERVER \\
\hline & & SYN, ACK & SMTP SERVER & GATEWAY \\
\hline & & ACK & GATEWAY & SMTP SERVER \\
\hline & & Ack & SMTP SERVER & MS \\
\hline & & 220 & SMTP SERVER & MS \\
\hline & & Ack & MS & SMTP SERVER \\
\hline & & EHLO & MS & SMTP SERVER \\
\hline & & Ack & SMTP SERVER & MS \\
\hline & & $\begin{array}{lr}250 & \text { HELO, } \\
\text { 8BITMIME, } & \text { SIZE, } \\
\text { DSN, AUTH } & \end{array}$ & SMTP SERVER & MS \\
\hline & & Ack & MS & SMTP SERVER \\
\hline & & AUTH & MS & SMTP SERVER \\
\hline & & Ack & SMTP SERVER & MS \\
\hline & & 334 & SMTP SERVER & MS \\
\hline & & $\begin{array}{l}\text {...authentication } \\
\text { challenge }\end{array}$ & SMTP SERVER & MS \\
\hline $\begin{array}{l}\text { STOP: SMTP: } \\
\text { Reply "235 } \\
\text { Authentication } \\
\text { successful" } \\
\text { received } \\
\text { by the client as an } \\
\text { answer to the } \\
\text { authentication } \\
\text { request. }\end{array}$ & & $\begin{array}{l}235 \text { Authentication } \\
\text { Successful }\end{array}$ & SMTP SERVER & MS \\
\hline
\end{tabular}

The process of E-mail login (upload) measurement can be started from by sending a synchronization signal from MS to Gateway until sending a 235 Authentication Successful signal from SMTP Server to MS. 
Table 11. Signaling Ladder Diagram of E-Mail Login (Upload)

\begin{tabular}{|c|c|c|c|c|}
\hline Trigger Point & $\begin{array}{l}\text { Logical } \\
\text { Channel }\end{array}$ & Signal Name & $\begin{array}{l}\text { Outgoing } \\
\text { Network } \\
\text { Element }\end{array}$ & $\begin{array}{l}\text { Incoming } \\
\text { Network } \\
\text { Element }\end{array}$ \\
\hline \multirow{37}{*}{$\begin{array}{l}\text { START: First TCP } \\
\text { "SYN" sent by the client }\end{array}$} & & SYN & MS & GATEWAY \\
\hline & & SYN, ACK & GATEWAY & MS \\
\hline & & ACK & MS & GATEWAY \\
\hline & & Connect & MS & GATEWAY \\
\hline & & DNS QUERY & GATEWAY & DNS SERVER \\
\hline & & DNS Response & DNS SERVER & GATEWAY \\
\hline & & SYN & GATEWAY & POP3 SERVER \\
\hline & & SYN, ACK & POP3 SERVER & GATEWAY \\
\hline & & ACK & GATEWAY & POP3 SERVER \\
\hline & & Connect & GATEWAY & POP3 SERVER \\
\hline & & Ack & POP3 SERVER & MS \\
\hline & & ${ }^{+} \mathrm{OK}$ & POP3 SERVER & MS \\
\hline & & Ack & MS & POP3 SERVER \\
\hline & & AUTH & MS & POP3 SERVER \\
\hline & & Ack & POP3 SERVER & MS \\
\hline & & ${ }^{+} \mathrm{OK}$ & POP3 SERVER & MS \\
\hline & & Ack & MS & POP3 SERVER \\
\hline & & AUTH & MS & POP3 SERVER \\
\hline & & Ack & POP3 SERVER & MS \\
\hline & & Authentication challenge & POP3 SERVER & MS \\
\hline & & ${ }^{+} \mathrm{OK}$ message, 1500 octets & POP3 SERVER & MS \\
\hline & & Ack & MS & POP3 SERVER \\
\hline & & STAT & MS & POP3 SERVER \\
\hline & & Ack & MS & POP3 SERVER \\
\hline & & ${ }^{+} \mathrm{OK} 11500$ & MS & POP3 SERVER \\
\hline & & Ack & MS & POP3 SERVER \\
\hline & & LIST & MS & POP3 SERVER \\
\hline & & Ack & POP3 SERVER & MS \\
\hline & & $\begin{array}{l}{ }^{+} \mathrm{OK} \text { Scan list follows } 1 \\
1500<\text { CRLF }>\text {. }<\text { CRLF }>\end{array}$ & POP3 SERVER & MS \\
\hline & & Ack & MS & POP3 SERVER \\
\hline & & UIDL & MS & POP3 SERVER \\
\hline & & Ack & POP3 SERVER & MS \\
\hline & & $\begin{array}{l}{ }^{+} \text {OK Scan list follows } 112 \\
<\text { CRLF }>\text { <CRLF }>\end{array}$ & POP3 SERVER & MS \\
\hline & & Ack & MS & POP3 SERVER \\
\hline & & RETR 1 & MS & POP3 SERVER \\
\hline & & Ack & POP3 SERVER & MS \\
\hline & & ${ }^{+} \mathrm{OK} 1500$ octets & POP3 SERVER & MS \\
\hline $\begin{array}{l}\text { STOP: Termination } \\
\text { sequence } \\
\text { <CRLF.CRLF> received } \\
\text { by the client as an } \\
\text { answer to the "RETR" } \\
\text { command. }\end{array}$ & & $\langle\mathrm{CRLF}\rangle,\langle\mathrm{CRLF}\rangle$ & POP3 SERVER & MS \\
\hline
\end{tabular}


The process of E-mail download session measurement can be started from by sending a synchronization signal from MS to Gateway until sending a $\langle\mathrm{CRLF}\rangle$. $\langle\mathrm{CRLF}\rangle$ signal from POP3 Server to MS.

Table 12. Signaling Ladder Diagram of E-Mail (Upload) Data Rate Measurement

\begin{tabular}{|c|c|c|c|c|}
\hline Trigger Point & $\begin{array}{l}\text { Logical } \\
\text { Channel }\end{array}$ & Signal Name & $\begin{array}{l}\text { Outgoing } \\
\text { Network } \\
\text { Element }\end{array}$ & $\begin{array}{l}\text { Incoming Network } \\
\text { Element }\end{array}$ \\
\hline \multirow[t]{14}{*}{$\begin{array}{l}\text { START: SMTP: } \\
\text { "MAIL FROM" sent by } \\
\text { the client }\end{array}$} & & $\begin{array}{l}\text { Mail from : } \\
\text { (name@domain.co } \\
\underline{\mathrm{m}}\end{array}$ & MS & SMTP SERVER \\
\hline & & Ack & MS & SMTP SERVER \\
\hline & & $250 \mathrm{OK}$ & SMTP SERVER & MS \\
\hline & & Ack & MS & SMTP SERVER \\
\hline & & RCPT TO & MS & SMTP SERVER \\
\hline & & Ack & SMTP SERVER & MS \\
\hline & & $250 \mathrm{OK}$ & SMTP SERVER & MS \\
\hline & & Ack & MS & SMTP SERVER \\
\hline & & DATA & MS & SMTP SERVER \\
\hline & & Ack & SMTP SERVER & MS \\
\hline & & $\begin{array}{l}354 \text { Start mail input; } \\
\text { end with } \\
\langle\mathrm{CRLF}\rangle .<\mathrm{CRLF}\rangle\end{array}$ & SMTP SERVER & MS \\
\hline & & Ack & MS & SMTP SERVER \\
\hline & & Message & MS & SMTP SERVER \\
\hline & & Ack & SMTP SERVER & MS \\
\hline $\begin{array}{l}\text { STOP: } \\
\text { SMTP: } \\
\text { Reply " } 250 \text { messages } \\
\text { accepted" received by the } \\
\text { client. }\end{array}$ & & $\begin{array}{l}250 \text { ok, message } \\
\text { accepted }\end{array}$ & SMTP SERVER & MS \\
\hline
\end{tabular}

The process of E-mail (upload) mean data rate measurement can be started from by sending a Mail from: (name@domain.com) signal from MS to SMTP Server until sending the $250 \mathrm{OK}$, message accepted signal from SMTP Server to MS.

Table 13. Signaling Ladder Diagram of E-Mail (Download) Data Rate measurement

\begin{tabular}{|l|l|l|l|l|}
\hline Trigger Point & $\begin{array}{l}\text { Logical } \\
\text { Channel }\end{array}$ & Signal Name & $\begin{array}{l}\text { Outgoing } \\
\text { Network } \\
\text { Element }\end{array}$ & $\begin{array}{l}\text { Incoming } \\
\text { network Element }\end{array}$ \\
\hline $\begin{array}{l}\text { Start: } \\
\text { POP3: } \\
\begin{array}{l}\text { RETR" command sent by the } \\
\text { client }\end{array}\end{array}$ & RETR 1 & MS & POP3 SERVER \\
\hline & & Ack & POP3 SERVER & MS \\
\hline $\begin{array}{l}\text { STOP: } \\
\text { POP3: } \\
\text { Termination sequence }\end{array}$ & OK 1500 octets & POP3 SERVER & MS \\
\hline
\end{tabular}


$\langle$ CRLF.CRLF $>$ received

by the client.

The process of E-mail (Download) mean data rate measurement can be started from by sending a RETR 1 signal from MS to POP3 Server until sending a $\langle\mathrm{CRLF}\rangle$. $\langle\mathrm{CRLF}\rangle$ signal from POP3 Server to MS.

\section{Note}

To the readers of this short paper, if you want to deepen the knowledge of this field, welcome to read the papers and books in the references.

\section{References}

[1] European Telecommunications Standards Institute. (2011). Speech and multimedia Transmission Quality (STQ), QoS aspects for popular services in mobile networks; Part 1. Assessment of Quality of Service, ETSI TS 102 250-1 V2.2.1

[2] European Telecommunications Standards Institute. (2011). ETSI Standard TS 102 250-2 v2.2.1: Speech and multimedia Transmission Quality (STQ); QoS aspects for popular services in mobile networks; Part 2: Definition of Quality of Service parameters and their computation.

[3] ETSI. (2008). ETSI Standard EG 202-057 V1.3.1: Speech Processing, Transmission and Quality Aspects (STQ).

[4] ITU-T. (2014). ITU-T Recommendation G.107: The E-Model, a computational model for use in transmission planning.

[5] Sigit Haryadi. (2013). Telecommunication Traffic: Technical and Business Consideration. Lantip Safari Media, Bandung, Indonesia. ISBN: 978-602-18578-5-4.

[6] Sigit Haryadi. (2013). Telecommunication Service and Experience Quality. Lantip Safari Media, Bandung, Indonesia. ISBN: 978-602-18578-6-1.

[7] Haryadi, Sigit; Limampauw, Ivantius. (2012). QoS Measurement of Telephony Services In 3G Networks Using Aggregation Method. Conference Proceeding of TSSA 2012. Denpasar, Indonesia.

[8] Haryadi, Sigit; Nusantara, Sandy. (2012). QoS Measurement of Web Browsing Services In 3G Networks Using Aggregation Method. Conference Proceeding of TSSA 2012. Denpasar, Indonesia.

[9] Haryadi, Sigit; Pramudita, Arnold. (2012). QoS Measurement of Video Streaming Services in a 3G Networks Using Aggregation Method. Conference Proceeding of TSSA 2012. Denpasar, Indonesia.

[10] Haryadi, Sigit; Andina, Raisha. (2012). QoS Measurement of File Transfer Protocol Services In 3 G Networks Using Aggregation Method. Denpasar, Indonesia. 2012.

[11] Haryadi, S. (2017, November). Probabilitas Statistik Untuk Insinyur. Researchgate. DOI10.13140/RG.2.2.14304.58885

[12] Sigit Haryadi. (2017). the Non-Intercept Linear Regression Method. Researchgate. DOI10.13140/RG.2.2.18721.71522

[13] [37] Sigit Haryadi. (2018, February). Applied Statistics for Assessment of the Regulation and Policy: case study in Telecommunication Industry. DOI10.13140/RG.2.2.15774.02884

[14] Haryadi, S. (2018, February 7). Statistik Terapan: Pengujian Regulasi \& Kebijakan Telekomunikasi. Retrieved from osf.io/3pkfw 
[15] Haryadi, S. (2018, January 25). Chapter 1. The Concept of

Telecommunication Network Performance and Quality of Service. Retrieved from osf.io/mukqb

[16] Haryadi, S. (2018, January 26). Chapter 2 of Network Performance and Quality of Service: Determination of Key Performance Indicator (KPI). Retrieved from osf.io/preprints/inarxiv/6gtnd

[17] Haryadi, S. (2018, January 26). Chapter 3 of Network Performance and Quality of Service: Technical Measurement of a Mobile Network Performance and Quality of Service. Retrieved from osf.io/q4wsz

[18] Haryadi, S. (2018, January 30). Basic Calculation of the Network's Availability and Reliability (Chapter 4 of Network Performance and Quality of Service). Retrieved from osf.io/preprints/inarxiv/z5mwq

[19] Haryadi, S. (2018, February 26). Measurement of Cellular Network's Availability and Reliability based on the Signaling Ladder Diagram. Retrieved from osf.io/preprints/inarxiv/hwvm8

[20] Haryadi, S. (2018, February 27). Performance Measurement of Telephone Network and Service. Retrieved from osf.io/preprints/inarxiv/5c7qa

[21] Haryadi, S. (2018, March 4). Performance Measurement of SMS and/or Chat Service. Retrieved from osf.io/preprints/inarxiv/h76uc

[22] Sigit Haryadi. (2017). Haryadi Index and Its Applications in Science of Law, Sociology, Economics, Statistics, and Telecommunications. Penerbit Elex Media Komputindo. Jakarta. ISBN 978-602-02-9895-5.

[23] Sigit Haryadi. (2017). Indeks Haryadi dan Penerapan di Ilmu Hukum, Sosiologi, Ekonomi, Statistik, dan Telekomunikasi. Penerbit Elex Media Komputindo, Jakarta. ISBN 978-602-02-9896-2

[24] Sigit Haryadi. (2016). Haryadi Index for Competition, Equality and Correlation Evaluation. Penerbit Lantip Safari Media, Bandung, Indonesia. ISBN 978-602-73231-3-2

[25] Sigit Haryadi. (2016). Haryadi Index untuk Evaluasi Kompetisi, Kesetaraan dan Korelasi. Penerbit Lantip Safari Media, Bandung, Indonesia. ISBN 978-60273231-3-1

[26] Sigit Haryadi. (2017). Calculation of the Mobile Communication Competition using Haryadi Index. DOI10.13140/RG.2.2.15634.25280

[27] [97] Sigit Haryadi. (2017). the Equality Correlation Method. Researchgate. DOI10.13140/RG.2.2.10443.80169.

[28] Muhammad Rizky Hikmatullah; Sigit Haryadi. (2017). Perceptual evaluation of speech quality over the top call service. 3rd International Conference on Wireless and Telematics (ICWT), Indonesia.

[29] Rizna Nofitasari; Sigit Haryadi. (2017). Self-healing mechanism with RSRP measurement in LTE network. 3rd International Conference on Wireless and Telematics (ICWT), Indonesia.

[30] Fidyatun Nisa; Sigit Haryadi. (2016). Simulation of the fault management with Self Healing mechanism (case study: LTE Network in Banda Aceh Area). 10th International Conference on Telecommunication Systems Services and Applications (TSSA), Indonesia.

[31] Suci Ramadona; Sigit Haryadi; Dyah Rakhma Aryanti. (2015). Over the top call service key performance indicator. 1st International Conference on Wireless and Telematics (ICWT), Indonesia. 
[32] Maya Rahayu; Sigit Haryadi; Dyah Rakhma Aryanti. (2015). Over the Top chat service key performance indicator. 1st International Conference on Wireless and Telematics (ICWT), Indonesia.

[33] Sigit Haryadi. (2016). Telecommunication Quality of Service Concept. Research gate. Available online at https://www.researchgate.net/publication/313101013_Telecommunication_Quality_of Service_Concept

[34] Sigit Haryadi. (2016). Grade of Service \& Quality of Service Concept and Analysis. Researchgate. Available online at https://www.researchgate.net/publication/313101070_Grade_of_Service_Quality_of Service_Concept_and_Analysis

[35] Sigit Haryadi. (2016). Performance Indicator of Telecommunication Service. Researchgate. Available online at https://www.researchgate.net/publication/313101244_Performance_Indicator_of_Tele communication_Service. 